\title{
INVERSE BREMSSTRAHLUNG ABSORPTION IN SPHERICAL LASER TARGETS
}

\author{
J. A. Nicolás and J. R. SanMartin \\ Escuela Técnica Superior de Ingenieros Aeronáuticos, Ciudad Universitaria, 28040 Madrid, Spain
}

(Received 7 August 1984; and in revised form 8 October 1984)

\begin{abstract}
Inverse bremsstrahlung has been incorporated into an analytical model of the expanding corona of a laser-irradiated spherical target. Absorption decreases slowly with increasing intensity, in agreement with some numerical simulations, and contrary to estimates from simple models in use up to now, which are optimistic at low values of intensity and very pessimistic at high values. Present results agree well with experimental data from many laboratories; substantial absorption $(>0.5)$ is found up to moderate intensities, say below $10^{15} \mathrm{~W} \mathrm{~cm}^{-2}$ for $1.06 \mu \mathrm{m}$ light. Anomalous absorption, when included in the analysis, leaves practically unaffected the abiation pressure and mass ablation rate, for given absorbed intensity. Universal results are given in dimensionless form.
\end{abstract}

\section{INTRODUCTION}

RECENT theoretical analyses of the spherical corona of a laser-irradiated pellet assumed that absorption occurs at the critical density $n_{c}$ (AFANAS'Ev et al., 1977; GITOMER et al., 1977; SANZ et al., 1981, hereafter referred to as Paper I; SANMARTIN et al., 1982; MAX et al., 1980; SANZ and SANMARTIN, 1983). Such a model is appropriate for small pellets and short, high-intensity, long wavelength, laser pulses. Inverse bremsstrahlung is then negligible and absorption is mainly due to processes at densities about critical.

The search for more ablative laser-target interaction has lately moved experiments into just the opposite conditions (MORA, 1982). Inverse bremsstrahlung absorption, which occurs at $n<n_{c}$, is then expected to be important. In the present paper we take this process into account, so as to correct and improve the results for ablation pressure, mass ablation rate, etc. obtained in Paper I. Those results were given in terms of the absorbed power $W$, not the incident power $W_{L}$, so that even when they are approximately correct, knowledge of the fractional absorption $A$ is helpful in using them. In this paper we calculate $A$ too.

The steady model of Paper I yielded dimensionless results as functions of $Z_{i}$ and $\hat{W} \equiv W / r_{a}^{2} \rho_{c} V^{3}$, where $V \equiv\left(r_{a} n_{c} / \bar{m}^{5 / 2} \bar{K}\right)^{1 / 4}$ is a convenient speed (SANMARTIN et al., 1982; SANZ and SANMartin, 1983); here $r_{a}$ is the ablation radius, $\bar{K}\left(Z_{i}\right)$ is Spitzer's heat-conductivity coefficient (SPITZER, 1967), $\rho_{c}$ is $\bar{m} n_{c}$, and $Z_{i}$ and $\bar{m} \equiv m_{i} / Z_{i}$ are ion charge-number and mass per unit-charge. In this paper we find that inverse bremsstrahlung introduces a third parameter, $\hat{V} \equiv \bar{m} V / m_{e} c$. This leads to modifications of scaling laws in some limits. For simplicity, we take $Z_{i}$ large in order to neglect ion pressure and internal energy.

The more ablative is the interaction (the lower $W / r_{a}^{2}$ and the higher $n_{c}$ or $r_{a}$ ), the lower is $\hat{W}$. Here we shall only consider the range $\hat{W}<10^{5}$. At higher powers, hotelectrons generated by resonant absorption (parametrized by $\hat{V}$ too) become important (SANMARTIN et al., 1982). At about $\hat{W}=10^{4}$ or $10^{5}$, heat-flux saturation effects (parametrized by the saturation or flux-limit factor $f$, and neglected here) become important too (MAX et al., 1980; SANZ and SANMARTIN, 1983). 
In Section 2 we present the equations of the model. Light is absorbed by inverse bremsstrahlung both on its way in and on its way out; for the sake of generality we let a fraction $\alpha$ of the power reaching the critical surface be absorbed there. The solution to the equations is discussed in Section 3. An asymptotic analysis for small $\hat{W}$ is given in Section 4 and Appendix. In Section 5 absorption results are discussed and compared with experimental data available. In Section 6 the dependence of ablation pressure $P_{a}$ and mass ablation rate $\dot{m}$ on $\hat{V}$ and $\alpha$ are discussed.

\section{EQUATIONS OF THE MODEL}

As in Paper I we use continuity, momentum, and energy equations for the ionelectron fluid

$$
\begin{gathered}
n v r^{2}=\mu \\
\bar{m} n v \mathrm{~d} v / \mathrm{d} r=-\mathrm{d}(n T) / \mathrm{d} r \\
4 \pi r^{2}\left(n v\left(\frac{1}{2} \bar{m} v^{2}+\frac{5}{2} T\right)-\bar{K} T^{5 / 2} \frac{\mathrm{d} T}{\mathrm{~d} r}\right)=\left(W_{i}-W_{r}\right) H\left(r-r_{c}\right)
\end{gathered}
$$

where $H$ is Heavyside's function. The energy equation has been modified to take into account that the power absorbed between the cold pellet surface at $r_{a}$, and any $r>r_{c}$, is the difference between the incident and reflected powers at $r$. The conductivity coefficient is just a function of $Z_{i}$ (SPITZER, 1967)

$$
\bar{K}\left(Z_{i}\right)=\varepsilon\left(Z_{i}\right) \delta_{T}\left(Z_{i}\right) 20(2 / \pi)^{3 / 2} / m_{e}^{1 / 2} e^{4} Z_{i} \ln \Lambda,
$$

if weak changes in the Coulomb logarithm are neglected.

Absorption on the incident and reflected rays is given by

$$
\begin{gathered}
\mathrm{d} W_{i} / \mathrm{d} r=\kappa W_{i} \\
\mathrm{~d} W_{r} / \mathrm{d} r=-\kappa W_{\mathrm{r}}
\end{gathered}
$$

where the absorption coefficient is (JoHNSTON and DAwsON, 1973)

$$
\kappa=\frac{320}{3 \pi} \varepsilon \delta_{T} \frac{\left(n / n_{c}\right)^{2}}{\left(1-n / n_{c}\right)^{1 / 2}} \frac{n_{c}}{\bar{K} m_{e} c T^{3 / 2}} .
$$

From (5) and (6) one gets $W_{i} W_{r}=(1-\alpha) W_{i c}^{2}, \alpha W_{i c}$ being the power absorbed at $r_{c}$. Then the right-hand side of (3) becomes

$$
W_{i}\left(1-(1-\alpha) W_{i c}^{2} / W_{i}^{2}\right) H\left(r-r_{c}\right)
$$

and $W_{r}$ drops off the analysis.

The dimensionless variables

$$
\begin{gathered}
\eta=r / r_{a}, \quad u=v /\left(T_{r} / \bar{m}\right)^{1 / 2} \\
\theta=T / T_{r}, \quad \bar{W}_{i}=W_{i} / 4 \pi \mu T_{r}
\end{gathered}
$$


lead to coupled equations for $u, \theta$ and $\bar{W}_{i}$

$$
\begin{gathered}
\frac{1}{2}\left(1-\frac{\theta}{u^{2}}\right) \frac{\mathrm{d} u^{2}}{\mathrm{~d} \eta}=\frac{2 \theta}{\eta}-\frac{\mathrm{d} \theta}{\mathrm{d} \eta} \\
\frac{1}{2} u^{2}+\frac{5}{2} \theta=\beta \eta^{2} \theta^{5 / 2} \frac{\mathrm{d} \theta}{\mathrm{d} \eta}+\bar{W}_{i}\left(1-(1-\alpha) \frac{\bar{W}_{i c}^{2}}{\bar{W}_{i}^{2}}\right) H\left(\eta-\eta_{c}\right) \\
\frac{\mathrm{d} \bar{W}_{i}}{\mathrm{~d} \eta}=\frac{320}{3 \pi} \frac{\varepsilon \delta_{T} \hat{V} u_{c}^{5 / 4} \eta_{c}^{5 / 2} \bar{W}_{i}}{\beta^{3 / 4} \eta^{4} u^{2} \theta^{3 / 2}\left(1-u_{c} \eta_{c}^{2} / u \eta^{2}\right)^{1 / 2}} ;
\end{gathered}
$$

we have $320 \varepsilon \delta_{T} / 3 \pi \simeq 10.6$ and 13.6 for $Z_{i}=16$ and $Z_{i} \rightarrow \infty$ respectively.

The boundary conditions are

$$
\begin{gathered}
\theta=0, u / \theta=1 \text { at } \eta=1 \\
\theta \rightarrow 0 \text { as } \eta \rightarrow \infty,
\end{gathered}
$$

and either one of two conditions

$$
\begin{gathered}
2 \theta / \eta=\mathrm{d} \theta / \mathrm{d} \eta \text { at } \eta_{s}, \\
\eta_{c}=\eta_{s},
\end{gathered}
$$

$\eta_{s}$ being the isothermal sonic point (where $u^{2}=\theta$ ); $T_{r}$ was chosen so as to have $u / \theta=1$ at $\eta=1$. Then

$$
\begin{gathered}
T_{r} / \bar{m} V^{2}=\beta^{1 / 2} \eta_{c} u_{c}^{1 / 2} \\
W_{L} / r_{a}^{2} \rho_{c} V^{3} \equiv \hat{W}_{L}=4 \pi \beta^{3 / 4} \eta_{c}^{7 / 2} u_{c}^{7 / 4} \bar{W}_{i x} \\
\dot{m} / 4 \pi r_{a}^{2} \rho_{c} V=\mu / r_{a}^{2} n_{c} V=\beta^{1 / 4} \eta_{c}^{5 / 2} u_{c}^{5 / 4} \\
P_{a} / \rho_{c} V^{2}=\beta^{1 / 2} \eta_{c}^{3} u_{c}^{3 / 2} .
\end{gathered}
$$

The total absorption and the absorption by inverse bremsstrahlung are given by

$$
\begin{gathered}
A_{T}=1-(1-\alpha) \bar{W}_{i c}^{2} / \bar{W}_{i \infty}^{2} \\
A_{B}=A_{T}-\alpha \bar{W}_{i c} / \bar{W}_{i \infty} .
\end{gathered}
$$

Considering $\alpha, \hat{V}$ and $\eta_{\mathrm{c}}$ (instead of $\hat{W}_{L}$ ) as free parameters, the four boundary conditions (11)-(13) suffice to solve the third-order system of differential equations (8)-(10) and determine the eigenvalue $\beta$. The dimensionless solution and equations (15)-(19) yield $r_{c} / r_{a}, \dot{m} / r_{a}^{2} \rho_{c} V, P_{a} / \rho_{c} V^{2}, A_{T}$ and $A_{B}$ as functions of $\hat{W}_{L}, \hat{V}$ and $\alpha$. We note that the results of Paper I are recovered by letting $\hat{V} \rightarrow 0$. 


\section{GENERAL INTEGRATION OF THE EQUATIONS}

The analysis of the overdense region is the same as that of Paper I. From (8) and (9) with $H=0$ one gets an equation $\mathrm{d} M^{2} / \mathrm{d} Y=F\left(M^{2}, Y\right)$ for $M^{2} \equiv u^{2} / \theta$ and $Y \equiv \beta \eta \theta^{5 / 2}$. This equation has a nodal point $M^{2}=0, Y=0$ (corresponding to $\eta=1$ ), where $Y \simeq \beta M^{5}$, and a saddle point $M^{2}=1, Y=3 / 2$ [corresponding to $\eta=\eta_{s}$ with condition (13a)]. If the sonic point lies in the overdense region $\left(\eta_{s}<\eta_{c}\right)$ one must use (13a), so that the solution is the integral curve crossing both singular points. This requires setting $\beta=\beta_{l} \simeq 11.3$; also, $\eta_{s}=\eta_{s l} \simeq 1.215$. Thus, for any $\eta_{c}>1.215$, one has both $u$ and $\theta$ in the range $1 \leqslant \eta \leqslant \eta_{c}$.

Since $\beta, u_{c}$ and $\theta_{c}$ are known, and $\bar{W}_{i c}$ is determined by condition (12), equations (8)-(10) may next be solved for the range $\eta_{c}<\eta<\infty$. As for $\hat{V}=0$, the temperature in the faraway plasma is found to behave as $\eta^{-2 / 5}$ for some specific $\hat{W}_{L}=\hat{W}_{L}^{*}(\hat{V}, \alpha)$, and $\eta^{-2 / 7}$ for $\hat{W}_{L}>\hat{W}_{L}^{*}$. Contrary to the $\hat{V}=0$ case, $\theta$ behaves as $\eta^{-6 / 5}$, not as $\eta^{-4 / 3}$, for $\hat{W}_{L}<\hat{W}_{L}^{*}$.

For fixed $\hat{V}$ and $x, \eta_{c}$ decreases with the power and reaches the value $\eta_{s l}$ at some particular $\hat{W}_{L}$. Both (13a) and (13b) are then satisfied; there are two slopes at $\eta_{c}$ and (13a) holds at $\eta_{c}^{-}$. For $\eta_{c}<\eta_{s l}$, equation (13b) must be used instead of (13a). This requires taking some $\beta<\beta_{l}$, and leads to $Y<3 / 2$ at $M^{2}=1$. To solve (8)-(10) for $\eta>\eta_{c}$ one proceeds as previously because $u_{s}$ and $\theta_{s}$ are already known, and $\eta_{c}=\eta_{s}$. Now the critical surface separates the subsonic and supersonic regions.

As $\eta_{c}$ goes on decreasing, a value $\eta_{c s}$ may be reached for which both (13a) and (13b) are again satisfied; (13a) holds at $\eta_{c}^{+}$. For $1<\eta_{c}<\eta_{c s}$, equation (13a) must be used again. Note that the underdense flow is first subsonic and then supersonic (SANZ and SANMARTIN, 1983).

Numerical results are discussed in Sections 5 and 6. Closed analytical results for low powers are obtained in the next section.

\section{DEFLAGRATION REGIMES $\left(\eta_{c} \rightarrow 1\right)$}

For low powers $\beta$ and $\eta_{c}-1$ are small. Then the conduction term of ( 9 ) is negligible except in a thin layer that encloses the critical surface, next to the pellet. The asymptotic analysis for low $\hat{W}_{L}$ is sensitive to the values of $\hat{V}$ and $\alpha$. Four parametric regimes may be distinguished as shown in Fig. 1.

We define for convenience a new parameter

$$
\sigma \equiv(320 / 3 \pi) \varepsilon \delta_{T} \hat{V} u_{c}^{5 / 4} / \beta^{3 / 4}
$$

and determine equations for $\sigma, \bar{W}_{i \infty}, \eta_{c}-1$, and $\bar{W}_{i c}$, which, together with (15) and (20), may yield $\beta, u_{c}, \bar{W}_{i \infty}, \bar{W}_{i c}$, and $\eta_{c}-1$ as functions of $\hat{W}_{L}, \hat{V}$ and $\alpha$; equations (16)-(19) then give the quantities of physical interest. We discuss the solutions outside and inside the thin deflagration layer (both overdense and underdense). Here we consider regimes I and II of Fig. 1 (which include the $\hat{V}=0$ (1) values of most interest), where $\bar{W}_{i c}, u_{c}$, and $\theta_{c}$ are small (for regimes III and IV see Appendix).

\section{a. Regimes I and II: outer solution}

Outside the thin layer, (9) and (10) become

$$
\frac{1}{2} u^{2}+\frac{5}{2} \theta=\bar{W}_{i}
$$




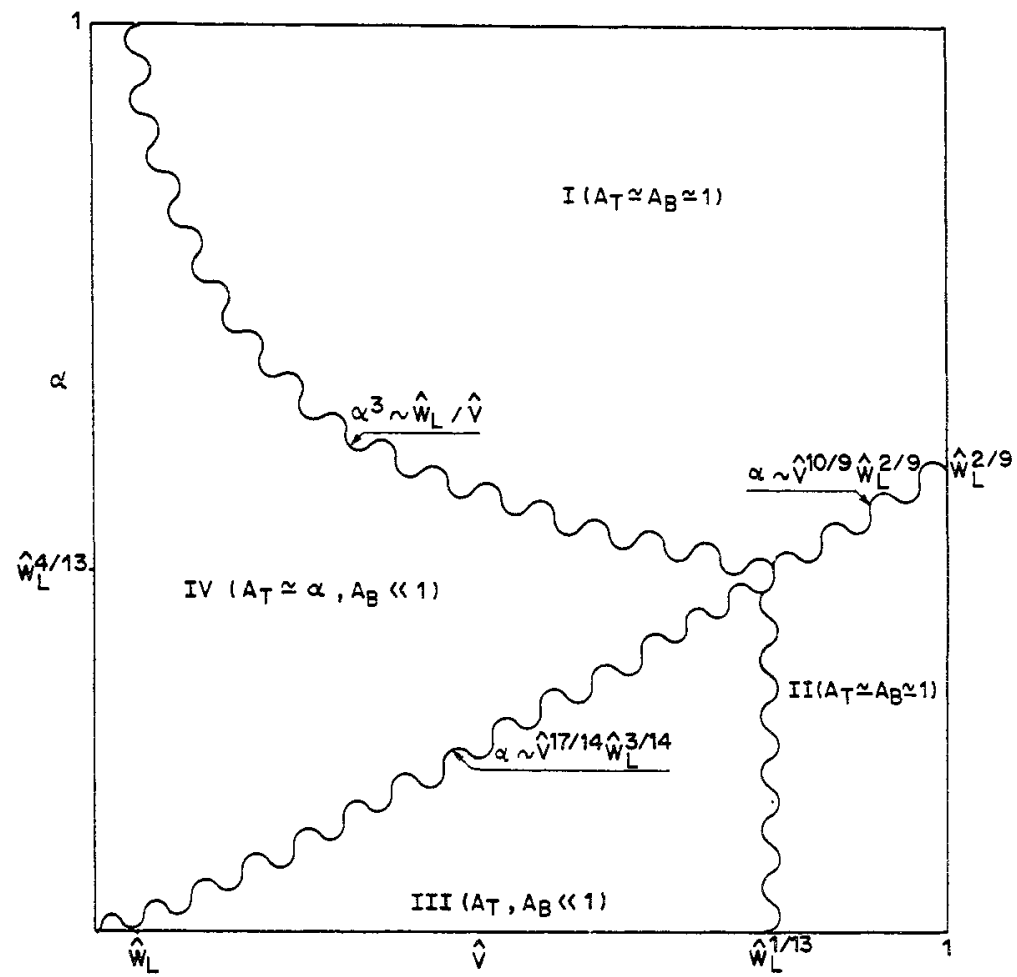

Fig. 1.-Schematic diagram showing the possible deflagration regimes, at low powers.

$$
\mathrm{d} \bar{W}_{i} / \mathrm{d} \eta=\sigma \bar{W}_{i} / \eta^{4} u^{2} \theta^{3 / 2}
$$

we have dropped the conduction term, and set $\eta_{c} \simeq 1, \bar{W}_{i c} / \bar{W}_{i} \simeq 0, u_{c} / u \simeq 0$. As later shown we have $u \simeq \theta$ throughout the inner solution so that both (12) and (11) hold as outer boundary conditions.

Using variables $M^{2} \equiv u^{2} / \theta$ and $\psi \equiv \eta^{3} \theta^{5 / 2} / \sigma,(8),(21)$, and (22) lead to a single equation

$$
\frac{\mathrm{d} \psi}{\mathrm{d} M^{2}}=\frac{\psi}{M^{2}} \frac{5\left(M^{2}-1\right)\left(M^{2}+5\right)-2 M^{2}\left(M^{2}+15\right) \psi}{2\left(M^{2}+5\right)\left(4 M^{2} \psi-M^{2}-1\right)}
$$

which has nodal points at $M^{2}=0, \psi=0$ (corresponding to the limit $\eta \rightarrow 1$ ); $\psi=0$, $M^{2} \rightarrow \infty ; M^{2}=0, \psi \rightarrow \infty$; and $\psi=5 / 2, M^{2} \rightarrow \infty$. There is also a saddle point at $\psi=2 / 5, M^{2}=5 / 3$ (conduction being negligible here, the sound speed is isentropic: $M_{s}^{2}=5 / 3$ ). The equation relating $\eta$ to $M^{2}$ and $\psi$ is

$$
\frac{\mathrm{d} M^{2}}{\mathrm{~d} \ln \eta}=\frac{\left(M^{2}+5\right)\left(4 M^{2} \psi-M^{2}-1\right)}{3 \psi\left(M^{2}-5 / 3\right)} .
$$

Figure 2 shows integral curves for (23). Curves labelled (i) lead to multivalued functions $M^{2}(\eta)$ at $M^{2}=5 / 3$; those labelled (ii) give $\theta \sim \eta^{2 / 7}$ for $\eta \rightarrow \infty$, violating (12). 


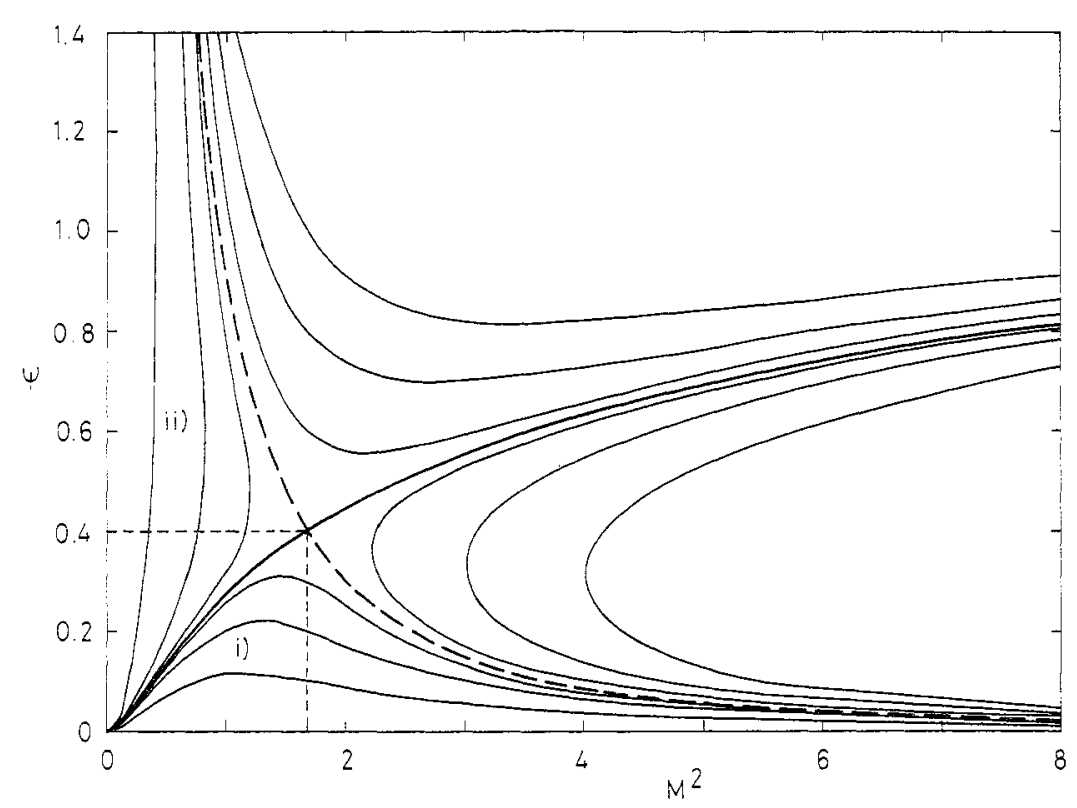

Fig. 2.-Integral curves for equation (23). The solution required is the curve through both the saddle point $M^{2}=5 / 3, \psi=2 / 5$, and the origin.

Hence, the solution is the curve joining the points $\psi=0, M^{2}=0$ and $\psi=2 / 5$, $M^{2}=5 / 3$. We then find

$$
\sigma \simeq 0.201, \bar{W}_{i \infty} \simeq 1.47
$$

we also find $\eta \simeq 1.15, \theta \simeq 0.310, \bar{W}_{i} \simeq 1.03$ at the sonic point.

b. Regimes I and II: overdense inner solution

Within the thin layer, we have $\mathrm{d} \theta / \mathrm{d} \eta \gg 2 \theta / \eta$. Using conditions (11), equation (8) becomes

$$
u=\theta+u^{2} \simeq \theta
$$

because both $\theta$ and $u$ are small. Equations (9) and (10) become

$$
\begin{gathered}
5 u / 2=\beta u^{5 / 2} \mathrm{~d} u / \mathrm{d} \eta+\bar{W}_{i}\left(1-(1-\alpha) \bar{W}_{i c}^{2} / \bar{W}_{i}^{2}\right) H \\
\mathrm{~d} \bar{W}_{i} / \mathrm{d} \eta=\sigma \bar{W}_{i} / \mathcal{u}^{3}\left(u-u_{c}\right)^{1 / 2} .
\end{gathered}
$$

For $\eta<\eta_{c}$, we have $H=0$; hence

$$
\begin{aligned}
& u^{5 / 2}=25(\eta-1) / 4 \beta, \\
& \eta_{c}-1=4 \beta u_{c}^{5 / 2} / 25 .
\end{aligned}
$$


c. Regime I: underdense inner solution

For $\eta>\eta_{c},(25)$ and (26) lead to

$$
\frac{5}{2} u-\bar{W}_{i}\left(1-(1-\alpha) \frac{\bar{W}_{i c}^{2}}{\bar{W}_{i}^{2}}\right)=\frac{\sigma \beta \bar{W}_{i}}{u^{1 / 2}\left(u-u_{c}\right)^{1 / 2}} \frac{\mathrm{d} u}{\mathrm{~d} \bar{W}_{i}},
$$

with the boundary condition $\bar{W}_{i}=\bar{W}_{i c}$ at $u=u_{c}$; for $\hat{W}_{L}, \hat{\nabla}$ and $\alpha$ given, only $\bar{W}_{i c}$ remains to be known. Now, to match the outer solution, the right-hand side of (28), arising from the conduction term in (25), should be negligible for $\eta-1 \gg \eta_{c}-1$. This implies, in turn, that conduction be negligible throughout, since otherwise $\mathrm{d} u / \mathrm{d} \bar{W}_{i}$ would be large near $\eta_{c}$, making impossible conduction to die off faraway. (We must have $\bar{W}_{i c} / u_{c}<0(1)$ for $\mathrm{d} u / \mathrm{d} \bar{W}_{i}$ to be positive as required.)

Thus, to lowest order we have

$$
u \simeq \frac{2}{5} \bar{W}_{i}\left(1-(1-\alpha) \bar{W}_{i c}^{2} / \bar{W}_{i}^{2}\right)
$$

For $\bar{W}_{i} \gg \bar{W}_{i c},(26)$ and (29) give $2 \bar{W}_{i} / 5 \simeq u \simeq(7 \sigma(\eta-1) / 2)^{2 / 7}$, matching the outer behavior at $\eta-1 \ll 1$. From (29) we get

$$
\bar{W}_{i c}=5 u_{c} / 2 \alpha
$$

equations (24), (27) and (30) determine our problem.

\section{d. Regime II: underdense inner solution}

To get $\bar{W}_{i c}$ to next order, we must study the neighborhood of $\eta_{c}$; simply using (29) on the right-hand side of (28) will not do, because of the factor $\left(u-u_{c}\right)^{1 / 2}$. Defining

$$
\begin{gathered}
\bar{W}_{i c}=(1-\varepsilon) 5 u_{c} / 2 \alpha, \quad \varepsilon \ll 1 \\
u / u_{c}=1+\varepsilon U, \quad \bar{W}_{i} / \bar{W}_{i c}=1+\varepsilon \alpha(\omega+1) /(2-\chi)
\end{gathered}
$$

(28) becomes

$$
\frac{\mathrm{d} U}{\mathrm{~d} \omega}=\frac{5 \alpha u_{\varepsilon} \varepsilon^{3 / 2}}{2 \sigma \beta(2-\alpha)} U^{1 / 2}(U-\omega)
$$

with the boundary condition $U=0$ at $\omega=-1$. Integral curves for (31) are shown in Fig. 3; the solution must be the separatrix, the only curve approaching $U-\omega=0$ as $\omega \rightarrow \infty$ (negligible conduction). Requiring it to cross the $\omega$-axis at $\omega=-1$ we find

$$
\bar{\varepsilon} \simeq 1.61\left(2 \sigma \beta(2-\alpha) / 5 \alpha u_{c}\right)^{2 / 3}
$$

Equation (30) is not valid if $\varepsilon=0(1)$, i.e. $\alpha u_{c} / \sigma \beta=0(1)$. Using (15), (20) and (24) this condition becomes $\alpha=0\left(\hat{V}^{10 / 9} \hat{W}_{L}^{2 / 9}\right)$ (see Fig. 1). For $\alpha$ of this order or lower, we define

$$
\frac{u}{u_{c}} \equiv \bar{U}, \frac{\bar{W}_{i}}{\bar{W}_{i c}} \equiv 1+\frac{5 u_{c}}{4 \bar{W}_{i c}} \bar{\omega}-\frac{\alpha}{2}
$$




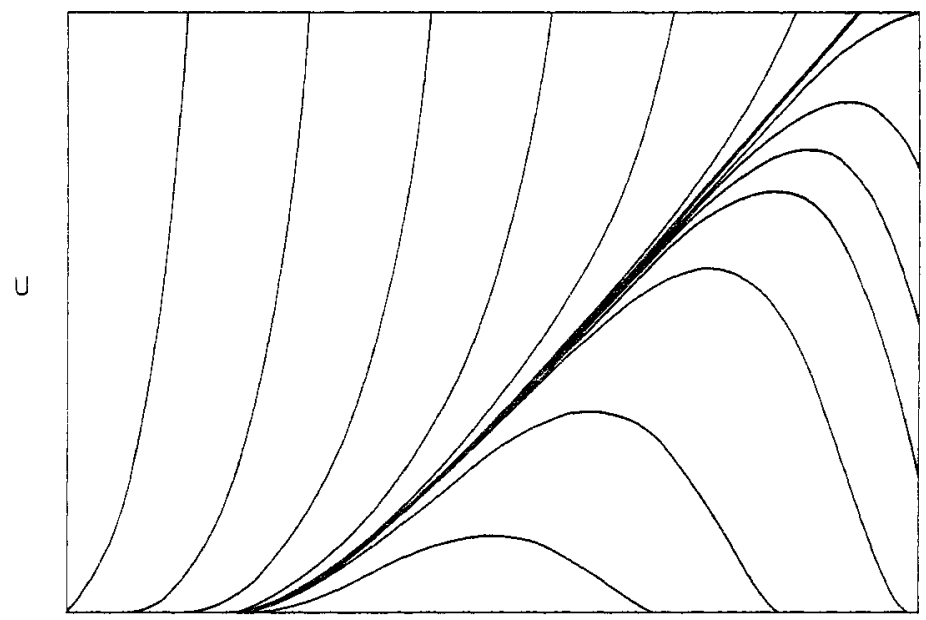

$\omega$

Fig. 3.-Integral curves (schematically) for equation (31). The required solution is the separatrix.

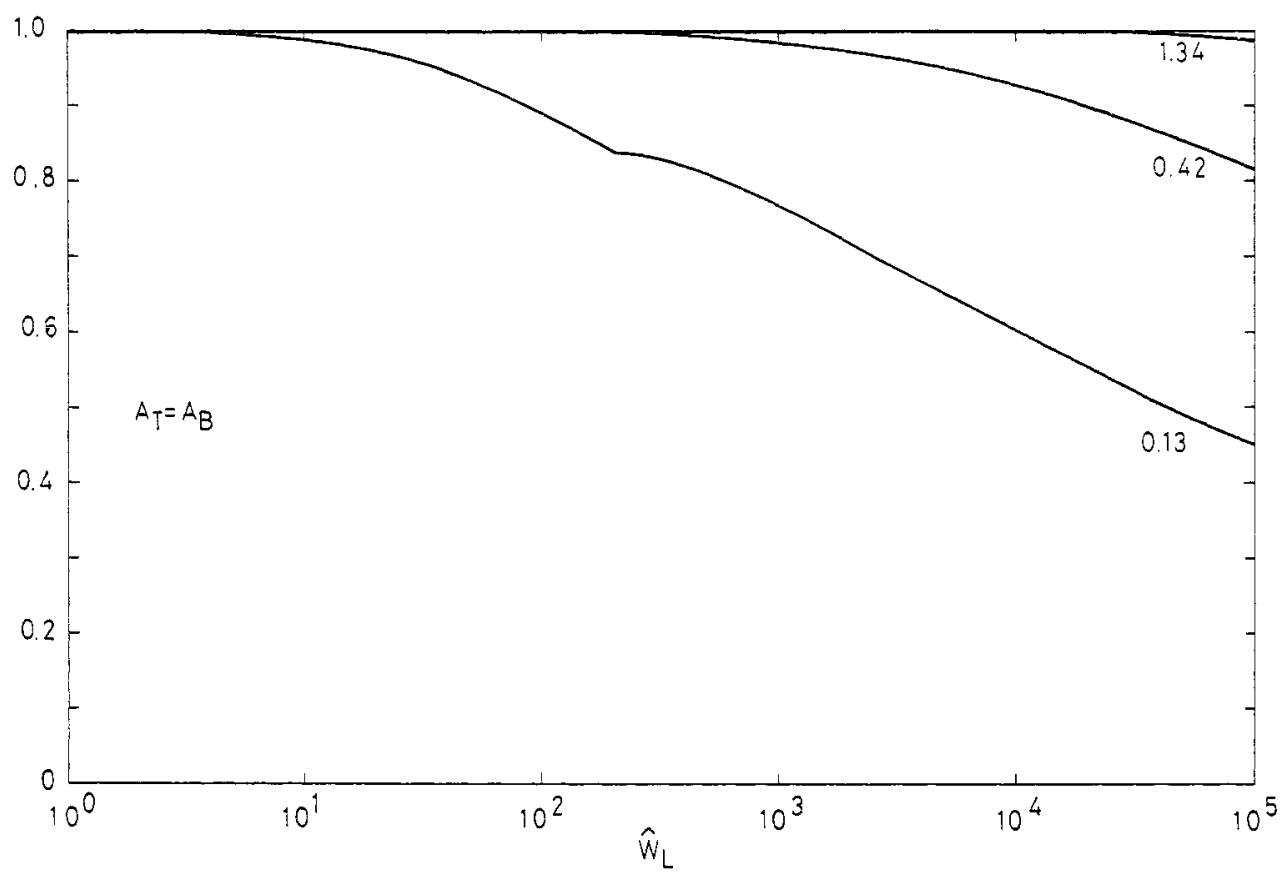

FIG. 4.- Total and bremsstrahlung absorption versus $\hat{W}_{L}$, for $\alpha=0$ and a few values of $\varepsilon\left(Z_{i}\right) \delta_{T}\left(Z_{i}\right) \hat{F} ; \hat{W}_{L}=W_{L} / r_{a}^{2} \rho_{c} V^{3}, \hat{V}=\bar{m} V / m_{e} c, V=\left(n_{c} r_{a} / \bar{m}^{5 / 2} \bar{K}\right)^{1 / 4}$.

then (28) becomes

$$
\frac{\mathrm{d} \bar{U}}{\mathrm{~d} \bar{\omega}}=\left(25 u_{c}^{2} / 8 \sigma \beta \bar{W}_{i c}\right) \bar{U}^{1 / 2}(\bar{U}-1)^{1 / 2}(\bar{U}-\bar{\omega})
$$


with the boundary condition

$$
\bar{\omega}=2 \alpha \bar{W}_{i c} / 5 u_{c} \text { at } \bar{U}=1 \text {. }
$$

Integral curves for (32) are similar to those in Fig. 3; the solution must be the (only) curve approaching the line $\bar{U}-\bar{\omega}=0$ for $\bar{\omega} \rightarrow \infty$. Use of (33) gives a relation between $u_{c}^{2} / \sigma \beta \bar{W}_{i c}$ and $\alpha \bar{W}_{i c} / u_{c}$, which we write

$$
\bar{W}_{i c}=\left(u_{c}^{2} / \sigma \beta\right) F_{1}\left(\alpha u_{c} / \sigma \beta\right)
$$

For $\alpha u_{c} / \sigma \beta \gg 1$ we have $F_{1} \simeq 5 \sigma \beta / 2 \alpha u_{c}$, and recover (30). For $\alpha u_{c} / \sigma \beta \ll 1$ (region II) we numerically obtain $F_{1} \simeq 1.67$ so that

$$
\bar{W}_{i c} \simeq 1.67 u_{c}^{2} / \sigma \beta
$$

\section{ABSORPTION RESULTS}

Figures 4 and 5 show total and bremsstrahlung absorption versus $\hat{W}_{L}$, for a few values of $\varepsilon \delta_{T} \hat{V}$, and $\alpha=0$ and 0.4 . Note that $\varepsilon \delta_{T} \hat{V} \propto n_{c}^{1 / 4} r_{a}^{1 / 4}, \hat{W}_{L} \propto W_{L} n_{c}^{-7 / 4} r_{a}^{-11 / 4}$. Since absorption is a growing function of both $\varepsilon \delta_{T} \hat{V}$ and $\hat{W}_{L}^{-1}$, it will increase if the power is reduced; it will increase strongly if either $r_{a}$ or $n_{c}$ is increased (or the wavelength $\dot{\lambda}$ is decreased).

Ion mass and charge effects are more complex. Note first that we cannot let $Z_{i} \rightarrow \infty$ (even though we assumed $Z_{i}$ large) because that implies $\bar{K} \rightarrow 0$. Accordingly, we retained $\varepsilon \delta_{T}$ as a function of $Z_{i}$ instead of setting it equal to $\varepsilon(\infty) \delta_{T}(\infty)(=0.4)$. Our

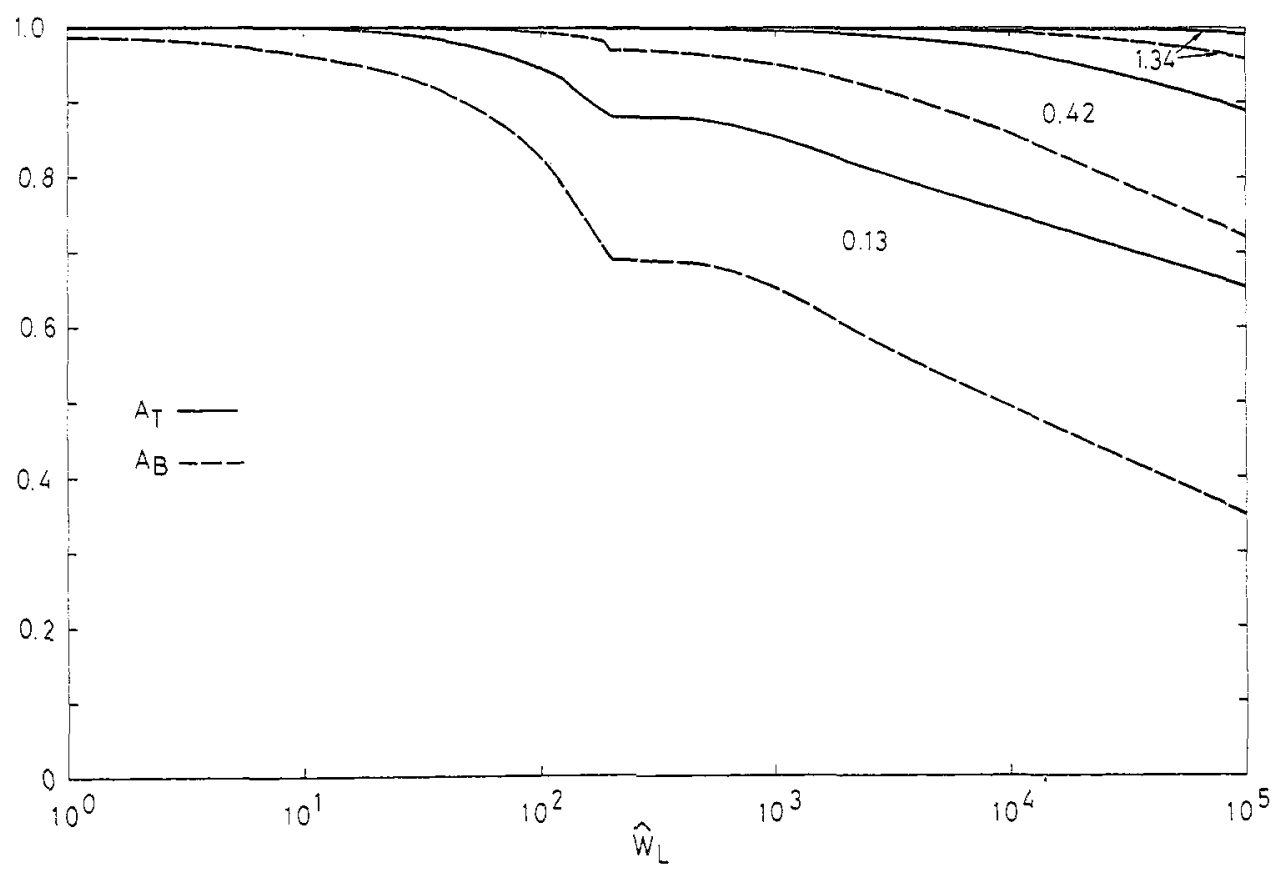

Fig. 5.-Same as Fig. 4, for $\alpha=0.4$. 


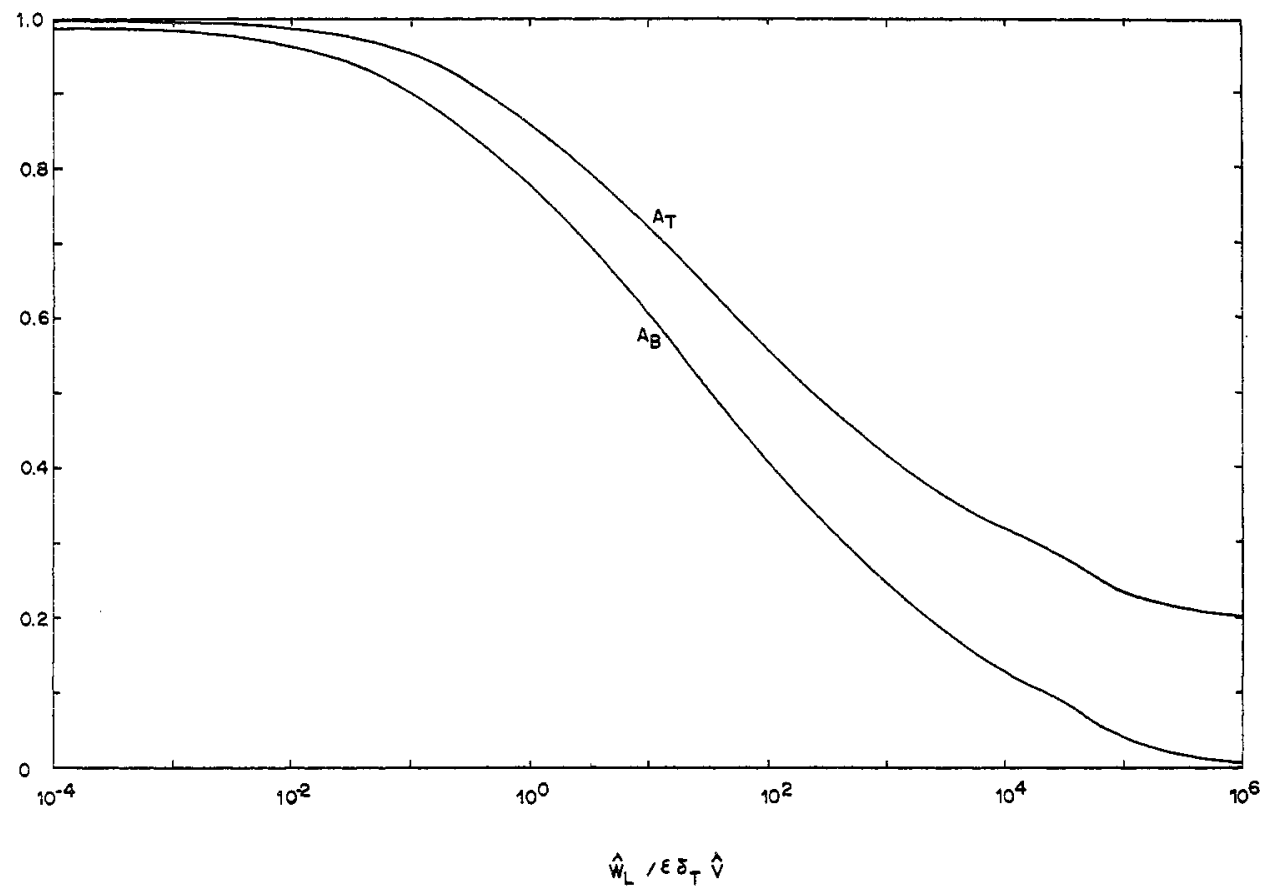

Fig. 6.-Total and bremsstrahlung absorption versus $\hat{W}_{L} / \varepsilon \delta_{T} \hat{V}$, for defiagration regimes I, IV; $\alpha=0.2$.

analysis should be reasonably valid for $Z_{i}$ not too low (SANZ and SANMARTIN, 1983).

At low or moderate mass number the corona will be fully ionized for all powers of interest, and the relations $Z_{i} \simeq m_{i} / 2 m_{p}, \bar{m} \simeq 2 m_{p}$ will hold. For $\bar{m}$ fixed both $\varepsilon \delta_{T} \hat{y}$ and $\hat{W}_{L}^{-1}$ grow with $Z_{i}$. Hence, for moderate $m_{i}$, absorption will increase with $m_{i}$. On the other hand ionization will not be complete at high mass number; we will have $Z_{i} \propto m_{i}^{b}, \bar{m} \propto \bar{m}^{1-b}$ with $b<1$. Although $\varepsilon \delta_{T} \hat{V}$ grows again with $m_{i}, \hat{W}_{L}^{-1}$ will only grow if $b>0.6$, roughly. Hence, without a model for ionization we cannot predict how increasing the mass number, when already high, will affect absorption. Note that for $m_{i}$ fixed and large, $Z_{i}$ will grow with the power; this leads to nearly constant ${ }_{\varepsilon} \delta_{T} \hat{V}$ and increasing $\hat{W}_{L}^{-1}$, so that absorption will grow.

At low powers the results from Section 4 and Appendix apply. Absorption now depends on just one parameter, aside from $\alpha$. For $\alpha$ not too small we have the deflagration regimes I, IV (Fig. 1); absorption is a function of $\alpha$ and $\hat{W}_{L} / \varepsilon \delta_{T} \hat{V}$, shown in Fig. 6 for $\alpha=0.2$. For $\alpha$ small enough (regimes II, III), absorption, shown in Fig. 7, depends on $\hat{W}_{L}^{1 / 13} / \varepsilon \delta_{T} \hat{V}$.

Figures 8,9 and 10 compare our absorption results, with data from experiments involving slab and disk targets, much more abundant than absorption data from experiments with pellets. The spherical analysis may be reasonably applied to planar experiments if the laser pulse is long and the spot small (MoRA, 1982). If the pulse is too short (for a given spot size) the corona has no time to evolve into a threedimensional, quasisteady form; it will be too short, so that the steady spherical model will overestimate the absorption. A spot too large (for a given pulse duration) has clearly a similar effect. 


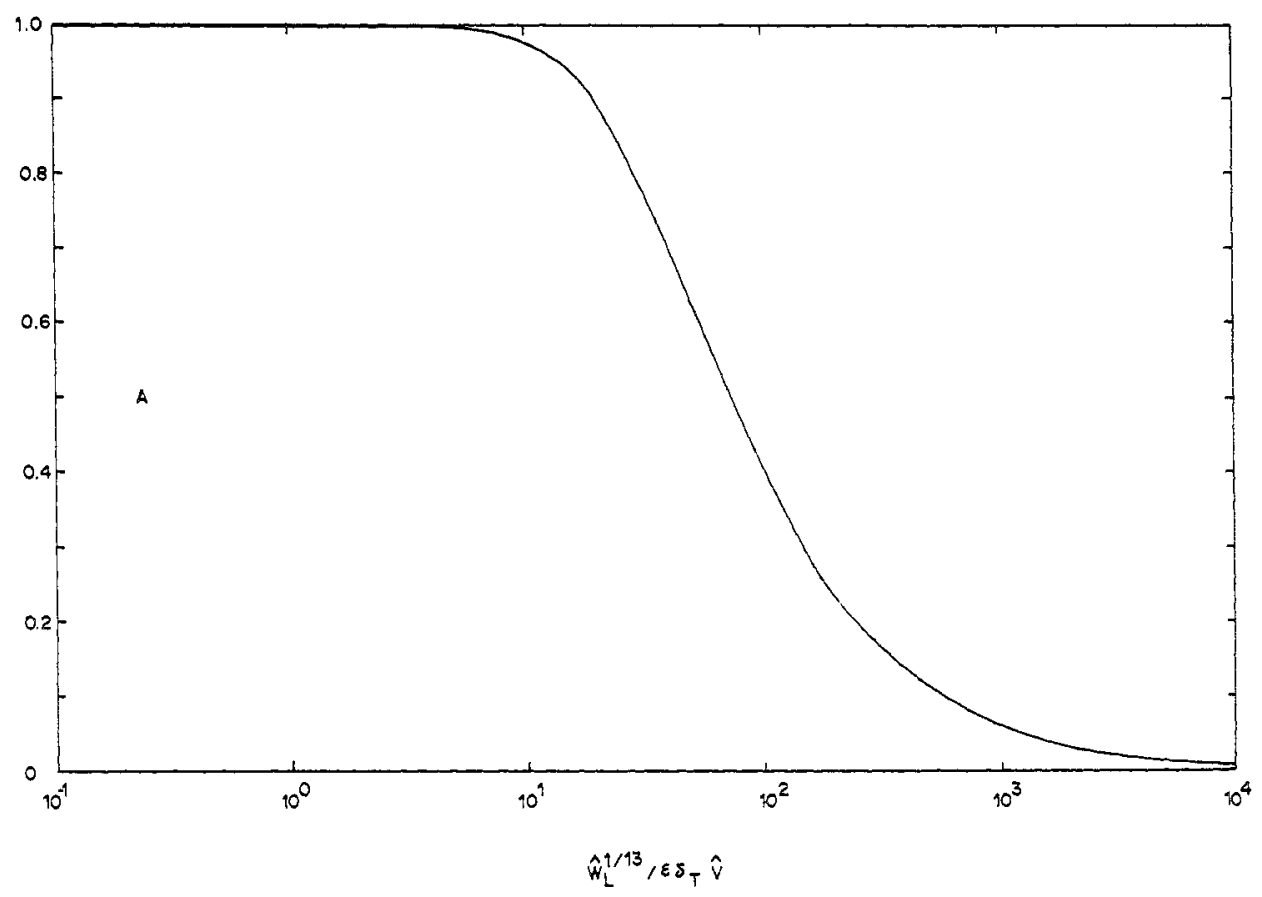

Fug. 7.-Total and bremsstrahlung absorption versus $\hat{W}_{L}^{1 / 13} / \varepsilon \delta_{T} \hat{\nabla}$, for defiagration regimes II, III: $\alpha \simeq 0$.

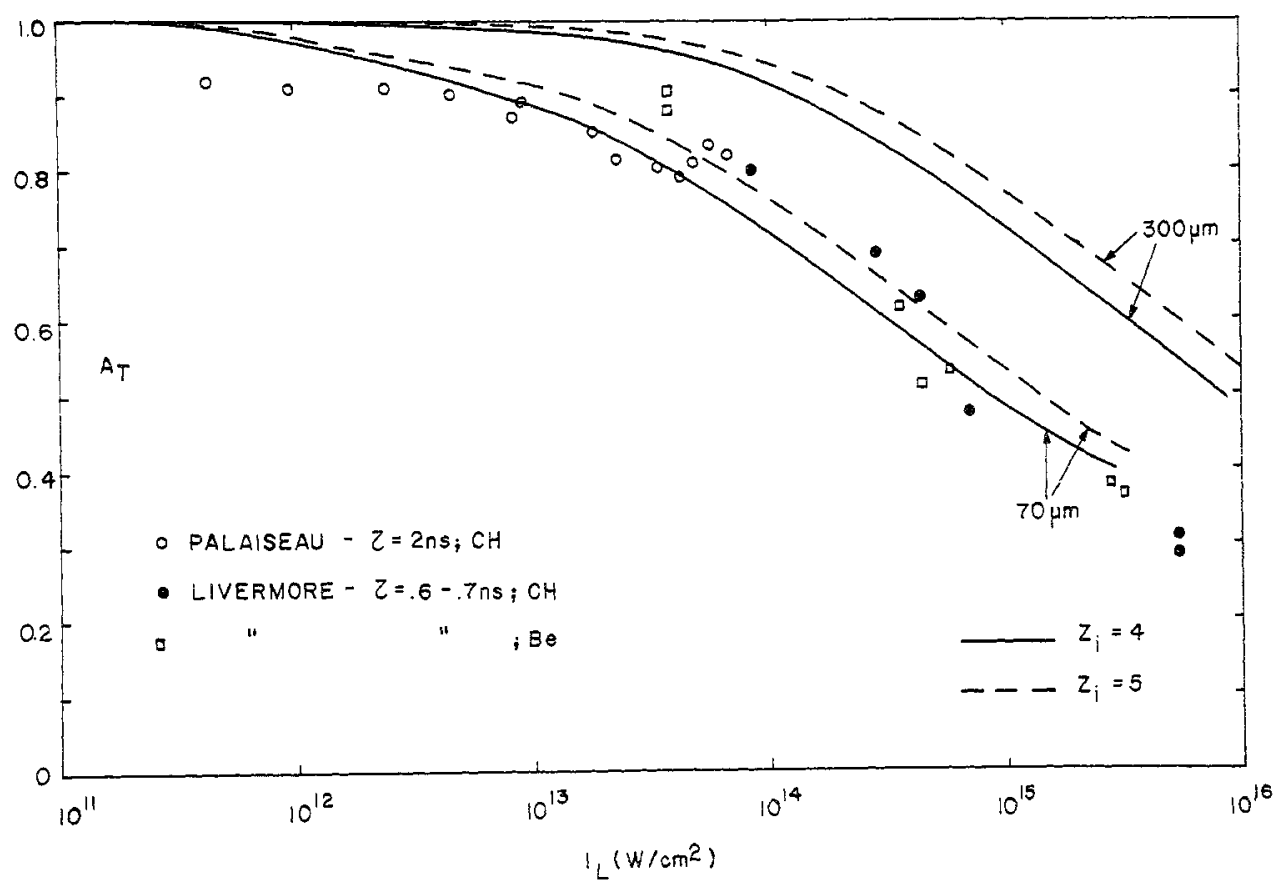

Fig. 8. - Total absorption versus incident laser intensity for $\lambda=0.53 \mu \mathrm{m}$. Experimental data are from planar $\mathrm{CH}$ and $\mathrm{Be}$ targets; $\tau$ is the puise length. Theoretical curves (adapted to planar geometry), have been drawn for $\alpha=0, Z_{i}=4$ and $5, \bar{m}=2 m_{p}, r_{a} \ln \Lambda=70$ and $300 \mu \mathrm{m}$. 


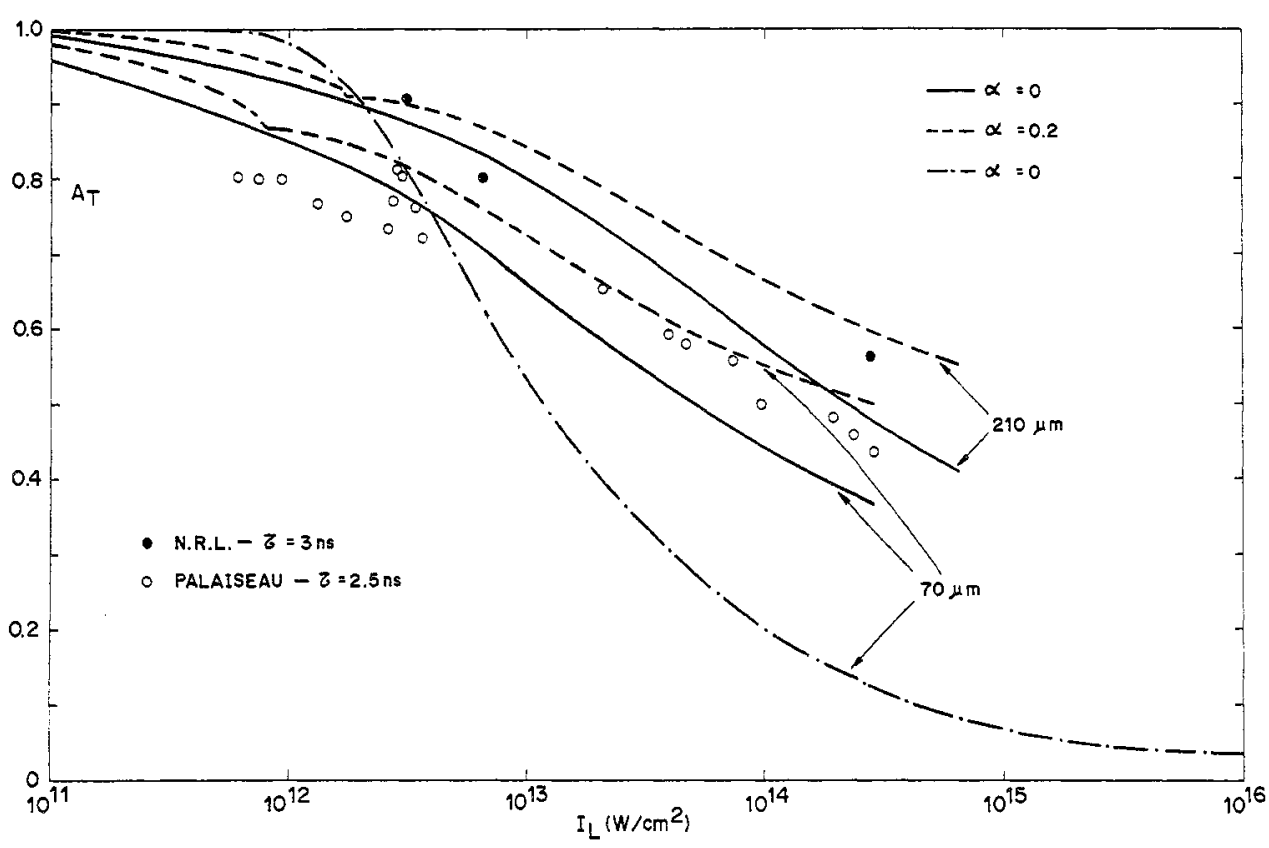

FIG. 9.-Same as Fig. 8, for $\lambda=1.06 \mu \mathrm{m}$ and $\mathrm{CH}$ targets. Theoretical curves drawn for $\alpha=0$ and $0.2, Z_{i}=5, \bar{m}=2 m_{p}, r_{a} \ln \Lambda=70$ and $210 \mu \mathrm{m}$. Also shown is the prediction (from an alternative model (MORA, 1982).

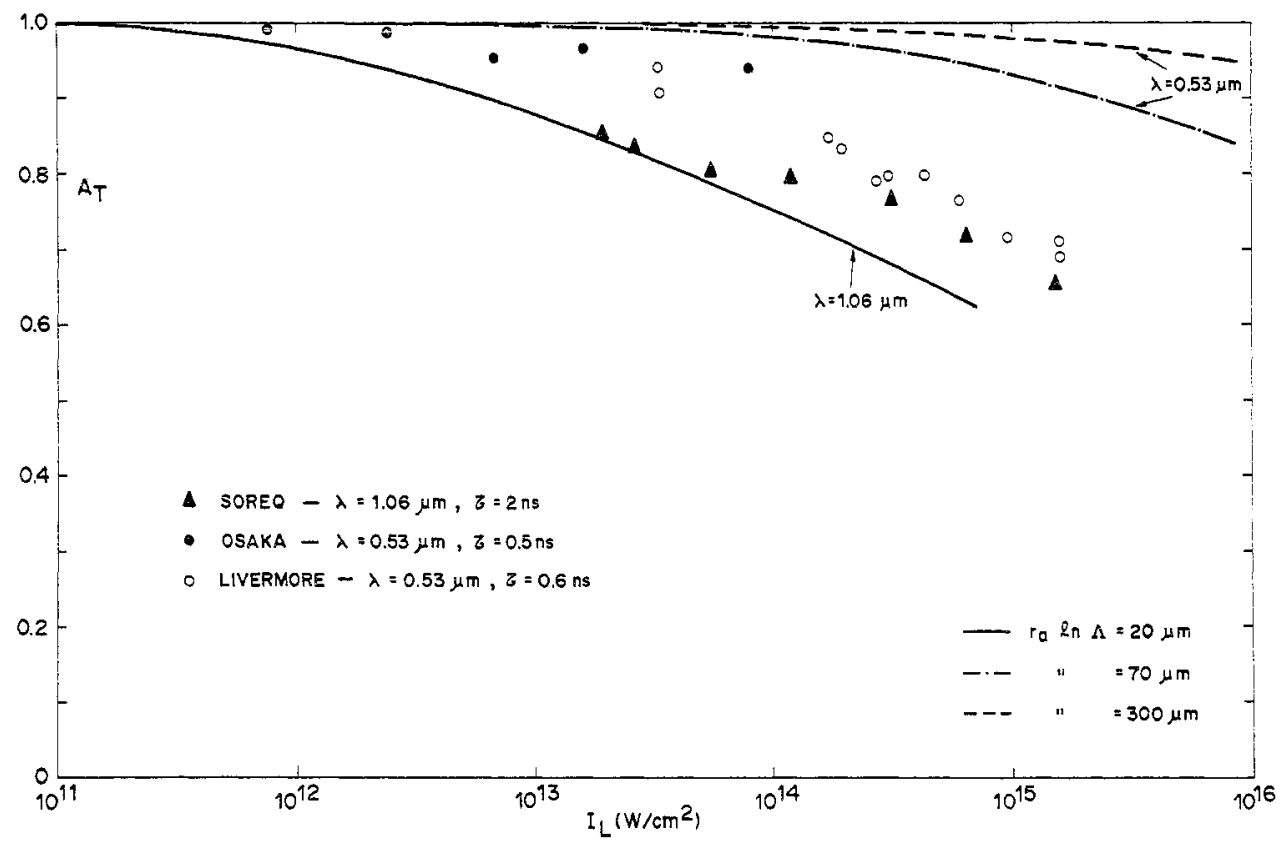

FIG. 10.-Same as Fig. 8, for $\lambda=0.53$ and $1.06 \mu \mathrm{m}$ and gold targets. Theoretical curves drawn for $\alpha=0, Z_{i}$ explained in the text, $\bar{m}=394 Z_{i}$. 
Figure 8 shows total absorption versus laser intensity $I_{L}$ from $\lambda=0.53 \mu \mathrm{m}$ experiments with Be and $\mathrm{CH}$ targets (GARBAN-LABAUNE et al., 1979; MEAD et al., 1983). To draw theoretical curves we used $W_{L} / 4 \pi r_{a}^{2} \eta_{c}^{2}$ for $I_{L}$. Since both $A_{T}\left(\hat{W}_{L}, \hat{\nabla}, \alpha\right)$ and $\eta_{c}\left(\hat{W}_{L}, \hat{V}, \alpha\right)$ are known from the analysis, we obtain

$$
A_{T}\left(I_{L}, \lambda, \alpha, \bar{m}, Z_{i}, r_{a} \ln \Lambda\right) .
$$

For fully ionized Be we have $\bar{m}=9 m_{p} / 4, Z_{i}=4$. For $\mathrm{CH}$ we have $n_{C}=n_{H}, n=6 n_{C}+n_{H}$ (quasineutrality), and $12 m_{p} n_{C}+m_{p} n_{H} \equiv \bar{m} n$ ( $\bar{m}$ enters the analysis through the inertia of the ions), leading to $\bar{m}=13 m_{p} / 7$; to estimate $Z_{i}$ we neglect the $8 \delta_{T}$ factor and get $36 n_{C}+n_{H} \equiv Z_{i} n\left(Z_{i}\right.$ only affects the collision frequency, for both conduction and absorption) leading to $Z_{i} \simeq 37 / 7$. We drew $A_{T}\left(I_{L}\right)$ for $\lambda=0.53 \mu \mathrm{m}, \alpha=0, \bar{m}=2 m_{p}$, and $Z_{i}=4$ and 5 . We also set $r_{a} \ln \Lambda=70$ and $300 \mu \mathrm{m}$. (Typically $\ln \Lambda \simeq 5$ or 6 . For $r_{a}$ we equate $r_{a}^{\frac{1}{2}}$ to spot radius, $\dagger$ which ranges from 20 to $75 \mu \mathrm{m}$ for most Livermore experiments; the maximum radius was $190 \mu \mathrm{m}$ ). For the smallest Livermore radii the agreement is good; theory slightly overestimates absorption probably because the pulse length ( $\tau \simeq 0.6,0.7 \mathrm{~ns}$ ) was a bit too short, for the corona to be quasisteady. For the largest radii, the corona was clearly unsteady: that is, the corona did not increase as the radius was increased; hence absorption should have been roughly independent of spot size, as found in the experiments indeed. The theoretical overestimate for the low- $I_{L}$ Ecole Politechnique data, is probably due to a large spot radius.

Figure 9 shows experimental (GARBAN-LABAUNE et al., 1979; RIPIN et al., 1980) and theoretical results for $\lambda=1.06 \mu \mathrm{m}$ light and $\mathrm{CH}$ targets. We drew curves for $\bar{m}=2 m_{p}, Z_{i}=5, \alpha=0$ and 0.2 (to show the effect of $\alpha$ ), and $r_{a} \ln \Lambda=70$ and $210 \mu \mathrm{m}$. There is agreement for the Naval Research Laboratory point at $I_{L}=7 \times 10^{14} \mathrm{~W} \mathrm{~cm}^{-2}(\ln \Lambda \simeq 6$, spot radius for $90 \%$ energy content $\simeq 50 \mu \mathrm{m}) ;$ the overestimate for the other two points is probably due to the large spot radii $(125$ and $225 \mu \mathrm{m})$. A similar explanation applies to the low-intensity points from Ecole Polytecnique.

For both our theory and the experiments, absorption decreases slowly with increasing intensity. This is in marked contrast with the fast decrease predicted by a simple model, repeatedly used in the past to estimate bremsstrahlung. Figure 9 shows $A_{T}\left(I_{L}\right)$ for one such model (MoRA, 1982) that uses a gross energy balance

$$
A_{T} I_{L}=4 n_{c} T_{c}^{3 / 2} / \bar{m}^{1 / 2}(\alpha=0)
$$

and an isothermal, exponential density profile of scale length $L$,

$$
A_{T}=1-\exp \left(-\frac{8}{3} \kappa^{*} L\right)
$$

where $\kappa^{*}=\kappa$ for $T=T_{c},\left(n / n_{c}\right)^{2}=\left(1-n / n_{c}\right)^{1 / 2}$; we set $\lambda=1.06 \mu \mathrm{m}, Z_{i}=5, \bar{m}=2 m_{p}$, $L \ln \Lambda=70 \mu \mathrm{m}$. An alternative model (HAAS et al., 1977; AHLSTROM, 1982) uses

$$
\begin{aligned}
A_{T} I_{L} & =f n_{c} T_{c}^{3 / 2} / m_{e}^{1 / 2} \\
A_{T} & =1-\exp \left(-\frac{32}{15} \kappa^{*} L\right)
\end{aligned}
$$

$\dagger$ We equate the spot area to half the area of a spherical target, $2 \pi r_{a}^{2}$. 


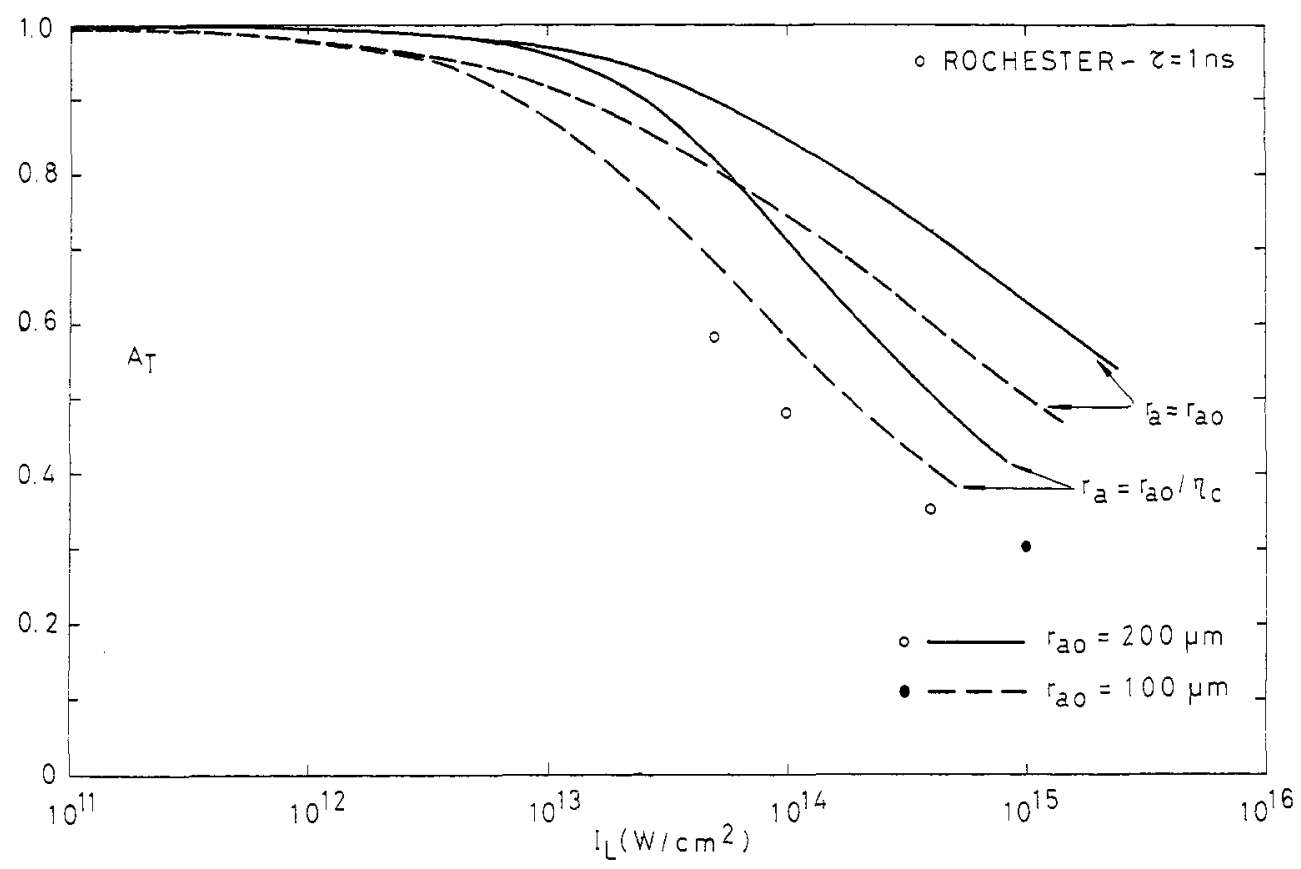

FIG. 11.-Same as Fig. 8; for $\lambda=1.06 \mu \mathrm{m}$ and spherical $\mathrm{CH}$ targets. Theoretical curves drawn for $\alpha=0, Z_{i}=5, \bar{m}=2 m_{p}, \ln \Lambda=6$, initial radius $r_{a 0}=100$ and $200 \mu \mathrm{m}$, and either final radius $r_{a}$ or final critical radius $r_{c} \equiv \eta_{c} r_{a}$, equal to $r_{a 0}$.

(linear density profile); the absorption may be obtained from Mora's curve by making the transformation $A_{T} \rightarrow A_{T}, I_{L} \rightarrow\left(f^{2} \bar{m} / 25 m_{e}\right)^{1 / 2} I_{L}$. These models are highly pessimistic at high intensities, and optimistic at low intensities. Note aiso that they give $A_{T}$ as a function of the ratio $\hat{W}_{L} / \hat{V}$, as in our analysis for the deflagration regimes I and IV.

Figure 10 shows experimental (Eliezer, 1982; MEAD et al., 1983; NishiMURA et al., 1983) and theoretical results for $\lambda=0.53$ and $1.06 \mu \mathrm{m}$; and gold targets. For such high atomic number the model can be suspected, since radiation transport and energy spent in ionization should affect the energy balance. As a rough approximation we set $Z_{i}=60\left(T_{c} / 1 \mathrm{keV}\right)^{1 / 2}$. The spot radius was 5,75 and $20-75 \mu \mathrm{m}$ for the Soreq, Osaka and Livermore experiments respectively.

Figure 11 compares our absorption results with experimental data for spherical $\mathrm{CH}$ targets (YAAKOBI et al., 1984) and $1.06 \mu \mathrm{m}$ light. The initial radius was $r_{a 0}=200$ $\mu \mathrm{m}$ for all data points except the highest intensity one $\left(r_{a 0}=100 \mu \mathrm{m}\right)$. To draw theoretical curves we set $\alpha=0, Z_{i}=5, \bar{m}=2 m_{p}, \ln \Lambda=6$ and either $r_{a}=r_{a 0}$ or $r_{c} \equiv \eta_{c} r_{a}=r_{a 0}$ (to roughly take into account target collapse). Theory overestimates absorption probably because $r_{a 0}$ is too large (the quasisteady approximation fails).

\section{OTHER RESULTS}

Figure 12 shows ablation pressure $P_{a}$ versus absorbed power. At low $\hat{W}$, bremsstrahlung effects decrease the pressure well below the $\vec{V} \rightarrow 0$ results of Paper I. One must go to unrealistically low values of $\hat{V}$ to approach that limit. At high powers and for the values of usual experiments $(\hat{V} \sim 1)$ the decrease is only about $20 \%$.

It is clear that in terms of absorbed power there can be no dependence on $\alpha$ at 


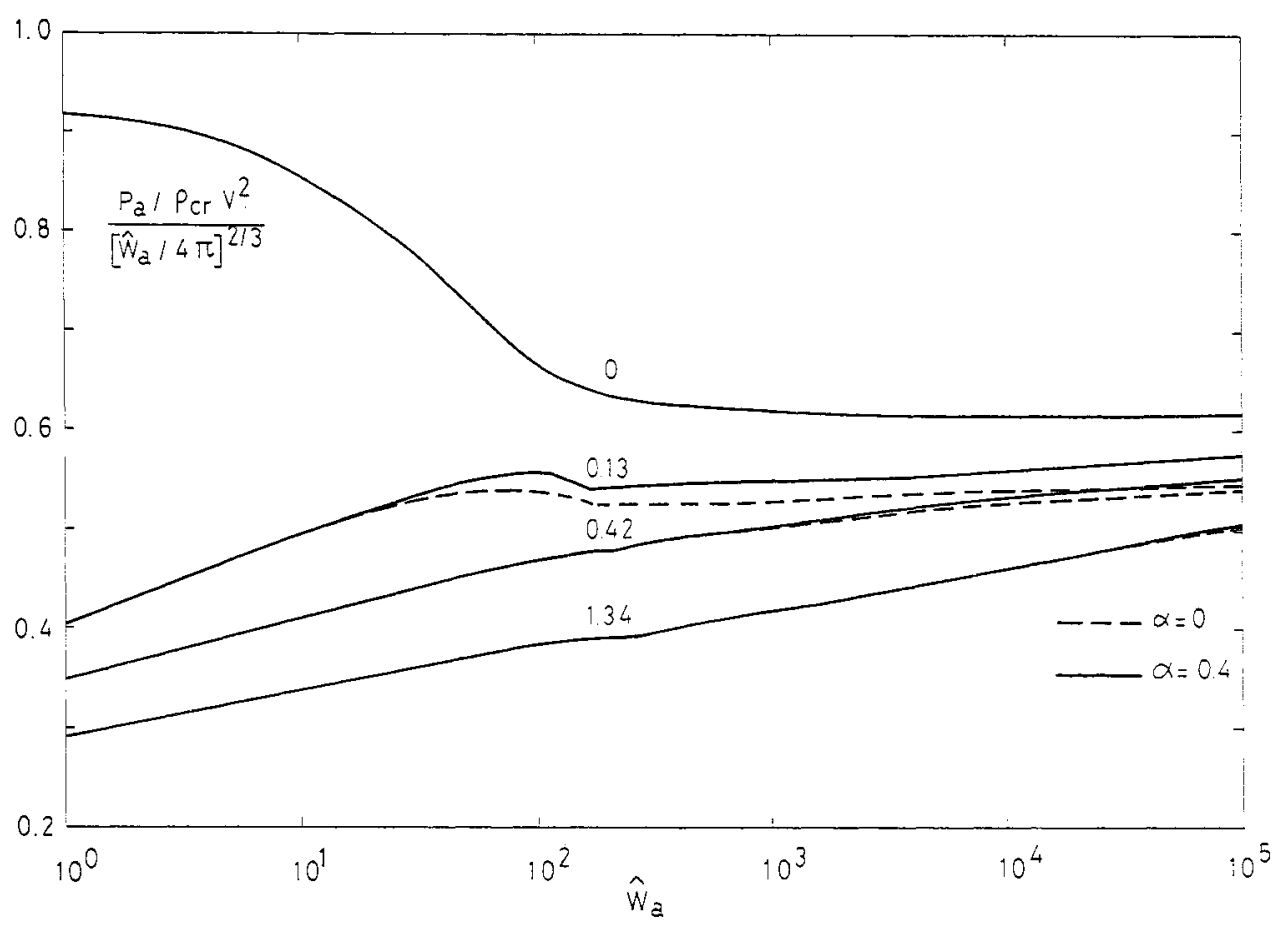

FIG. 12.-Normalized ablation pressure versus absorbed power, for several values of $\varepsilon \bar{\delta}_{T} \hat{V}$ and $\alpha=0$ and 0.4 .

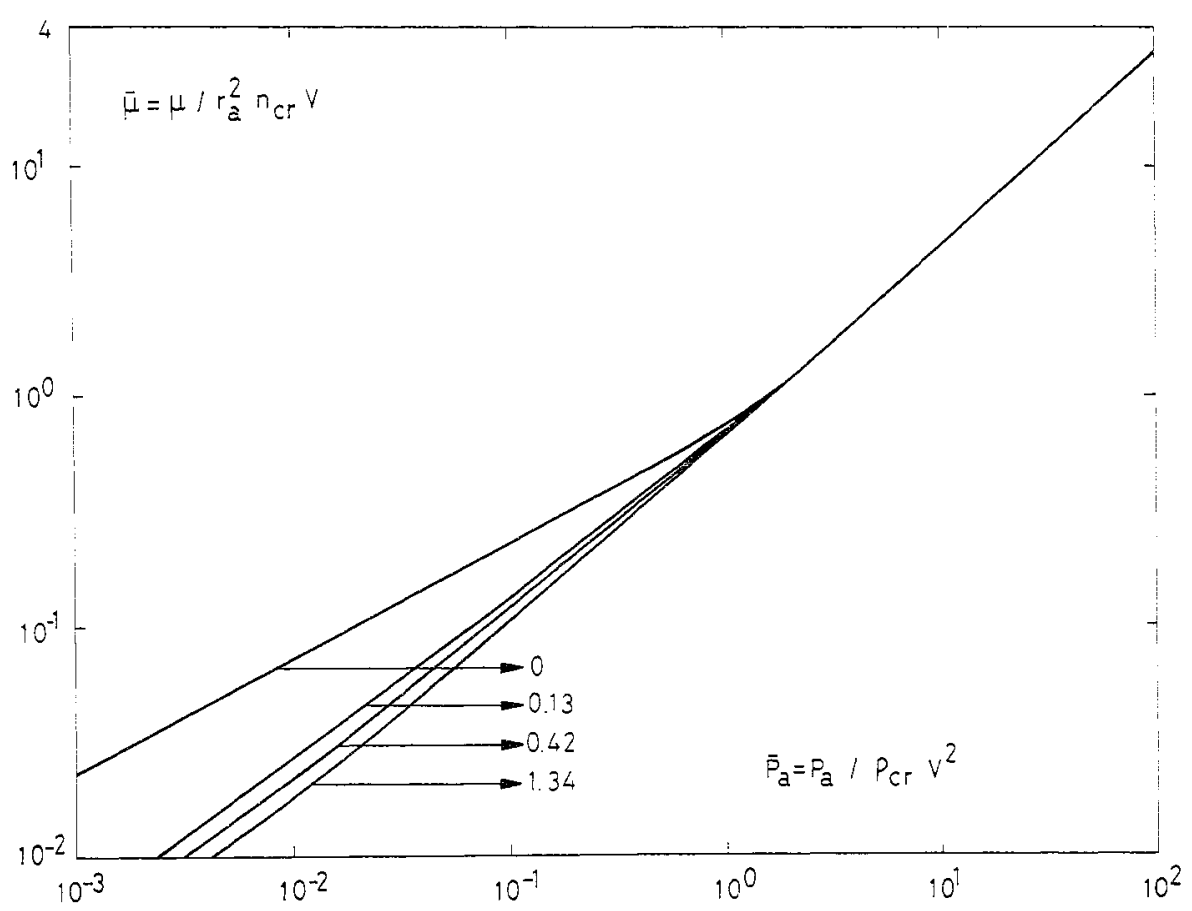

Fig. 13.-Dimensionless mass ablation rate versus ablation pressure, for several values of $\varepsilon \delta_{T} \hat{V}$ and any $x$. 
high or lower powers, for quantities other than absorption. At high powers inverse bremsstrahlung is inefficient, all energy being absorbed at the critical surface; at low powers almost no energy reaches that surface, so that anomalous absorption is nil. Figure 12 goes further, showing that the dependence on $\alpha$ is very weak at intermediate powers too.

Figure 13 shows mass ablation rate $\dot{m} \equiv 4 \pi m_{i} \mu / Z_{i}$ versus $P_{a}$. Above some power the flow at the critical surface is supersonic and there is no dependence on $\hat{V}$; at low powers and typical values of $\hat{V}, \dot{m}$ lies well below the $\hat{V} \rightarrow 0$ limit. There is no dependence on $\alpha$.

\section{DISCUSSION}

Simple models of inverse bremsstrahlung for laser irradiated targets (AHLSTROM, 1982; MORA, 1982) predict negligible (less than 0.1) absorption at moderate intensities: say $I \lesssim 10^{15} \mathrm{~W} \mathrm{~cm}^{-2}$ for $\lambda=1.06 \mu \mathrm{m}$. Experimental values lie around 0.5 . In the present paper we incorporate inverse bremsstrahlung into a quasisteady model for spherical targets (SANZ et al., 1981; SANZ and SANMARTIN, 1983). We obtain absorption theoretical results and compare them with experimental data; the agreement is good. The basic novel feature, also found in some numerical simulations (CRAXTON and MCCRORY, 1980; MEAD et al, 1982), is that absorption decreases slowly with increasing I, contrary to the behavior of simple models, which are optimistic at low intensities and very pessimistic at high intensities.

The model determines all dimensionless quantities (such as fractional absorption $\mathrm{A}$ ) as functions of four parameters: $Z_{i}$, the anomalous fractional absorption $\alpha$, $\hat{W}_{L} \equiv W_{L} / r_{a}^{2} \rho_{c} V^{3}$, and $\hat{V} \equiv \bar{m} V / m_{e} c$, where $V \equiv\left(n_{c} r_{a} / \bar{m}^{5 / 2} \bar{K}\right)^{1 / 4}$. In this paper we neglected ion pressure and internal energy (large $Z_{i}$ approximation; finite $Z_{i}$ effects have been studied elsewhere (SANZ and SANMARTIN, 1983)).

We have

$$
\begin{aligned}
\hat{W}_{L} & \simeq \frac{4.56 W_{L}}{10^{10} \text { watts }}\left(\frac{A_{i}}{2 Z_{i}}\right)^{7 / 8}\left(\frac{\lambda}{1.06 \mu \mathrm{m}}\right)^{7 / 2}\left(\frac{1 \mathrm{~mm}}{2 r_{a}}\right)^{11 / 4}\left(\frac{100 \varepsilon \delta_{T}}{Z_{i} \ln \Lambda}\right)^{3 / 4} \\
\hat{V} & \simeq 1.71\left(\frac{A_{i}}{2 Z_{i}}\right)^{3 / 8}\left(\frac{1.06 \mu \mathrm{m}}{\lambda}\right)^{1 / 2}\left(\frac{2 r_{a}}{1 \mathrm{~mm}} \frac{Z_{i} \ln \Lambda}{100 \varepsilon \delta_{T}}\right)^{1 / 4} .
\end{aligned}
$$

Inverse bremsstrahlung is parametrized by $\hat{V}$, and vanishes when $\hat{V} \rightarrow 0$. Only the "ablative" regime has been considered, roughly $\hat{W}_{L}<10^{5}$; above $10^{5}$, saturated flux and hot-electron effects (parametrized by $\hat{V}$ too) become important (SANMARTIN et al., 1982; SANZ and SANMARTIN, 1983).

At low powers the flow at the critical surface is subsonic (as in the finite $Z_{i}$ case). At very low powers $\left(\hat{W}_{L}<1\right)$ a thin deflagration layer appears next to the pellet surface, and outside it conduction is negligible. In terms of absorbed power quantities other than absorption are practically independent of $\alpha$. The dependence on $\hat{V}$ is substantial at low powers, and weak at high ones. 


\section{APPENDIX}

\section{a. Deflagration regime III}

The analysis of regime II fails when $u_{c}^{2} / \sigma \beta \sim \bar{W}_{i c} \geqslant 0(1)$, or [by using (15), (20) and (24)] $\hat{W}_{L} / \hat{V}^{13} \geqslant 0(1)$ (Fig. 1). Sections $4 b$ and $d$, and in particular equátions (27) and (34), need no modification; on the other hand the outer solution requires replacing (21) by the equation

$$
\frac{1}{2} u^{2}+\frac{5}{2} \theta=\bar{W}_{i}\left(1-\bar{W}_{i c}^{2} / \bar{W}_{i}^{2}\right)
$$

Though system (8), (22) and (A.1) cannot be reduced to a single equation as in Section $4 a$, it is easy to show that if its solution is to be univalued at the isentropic sonic point we must have

$$
\theta^{s / 2} \eta^{3} / \sigma=2\left(\bar{W}_{i}^{2}+\bar{W}_{i c}^{2}\right) / 5\left(\bar{W}_{i}^{2}-\bar{W}_{i c}^{2}\right) \text { at } u^{2}=5 \theta / 3 .
$$

With this condition the system may be solved, and $\sigma$ and $\bar{W}_{i x}$ determined for any given $\bar{W}_{i c} ;$ thus, we get

$$
\sigma=F_{2}\left(\bar{W}_{i c}\right), \quad \bar{W}_{i \infty}=F_{3}\left(\bar{W}_{i c}\right) .
$$

For $\bar{W}_{\text {ic }}$ small we have $F_{2} \rightarrow 0.201, F_{3} \rightarrow 1.47$ (regime II); for $\bar{W}_{i c}$ large $\left(\hat{V} \ll \hat{W}_{L}^{1 / 13}\right.$ ) we numerically get

$$
\sigma \simeq 0.075 / \bar{W}_{i c}, \quad \bar{W}_{i \infty} \simeq \bar{W}_{i c} .
$$

\section{b. Deflagration regime IV}

The analysis of regime I fails when $\bar{W}_{i c} \geqslant 0(1)$ too; here, this condition is equivalent to $u_{c} / \alpha \geqslant 0(1)$ or $\hat{W}_{L} / \alpha^{3} \hat{D} \geqslant 0(1)$. For $\alpha$ small we still have $u_{c} \simeq \theta_{c} \ll 1$ so that Section $4 \mathrm{~b}$ needs no modification. The outer and inner underdense solutions are those of regimes III and I respectively, so that equations (27), (30) and (A.2) apply. This same conclusion may be reached by looking at the transition from III to IV (Fig. 1).

For $\alpha=0(1), \bar{W}_{i c}, u_{c}$ and $\theta_{c}$ become simultaneously of order unity, so that both the inner and outer analysis of Section 4 break down. The inner solution is now given by

$$
\begin{gathered}
u=\theta+u^{2} \\
\frac{1}{2} u^{2}+\frac{5}{2} \theta=\beta \theta^{5 / 2} \frac{\mathrm{d} \theta}{\mathrm{d} \eta}+\bar{W}_{i}\left(1-(1-\alpha) \frac{\bar{W}_{i c}^{2}}{\bar{W}_{i}^{2}}\right) H \\
\mathrm{~d} \bar{W}_{i} / \mathrm{d} \eta=\sigma \bar{W}_{i / u^{3 / 2}} \theta^{3 / 2}\left(u-u_{c}\right)^{1 / 2} .
\end{gathered}
$$

For $\eta<\eta_{c}$, using (A.3) in (A.4) leads to an expression for $\eta(u)$. In particular we get

$$
\eta_{c}-1=2 \beta \int_{0}^{u_{c}} \frac{(1-2 u)(1-u)^{5 / 2} u^{3 / 2}}{5-4 u} \mathrm{~d} u .
$$

Note that we must have $u_{c}<1 / 2$; otherwise $u(\eta)$ would become multivalued at $u=1 / 2$.

For $\eta>\eta_{c},\left(\right.$ A.3) shows that $d \bar{W}_{i} / \mathrm{d} \eta \leqslant 0(1)$ for $\sigma \leqslant 0(1)$; thus we have $\bar{W}_{i} \simeq$ const $=\bar{W}_{i c}$ throughout the underdense layer. From (A.4) and (A.5) we get

$$
\frac{\mathrm{d} u}{\mathrm{~d} \eta}=\frac{\frac{5}{2} u-2 u^{2}-\alpha \bar{W}_{i c}}{\beta u^{5 / 2}(1-u)^{5 / 2}(1-2 u)} .
$$

The numerator of (A.7) (a) must have the sign of $(1-2 u$ ), (b) grows with $u$ for $u<5 / 8$, (c) must vanish for $\eta-1 \gg \eta_{c}-1$. Two cases may then be distinguished: If $u_{c}<1 / 2$ we have $u=$ const $=u_{c}, \theta=$ const $=\theta_{c}$; therefore

$$
\frac{5}{2} u_{c}-2 u_{c}^{2}=\alpha \bar{W}_{i c}, \text { if } 0<\alpha \bar{W}_{i c}<3 / 4
$$


If $u_{c}=1 / 2,(\mathrm{~A} .7)$ gives $u(\eta)$ growing from $1 / 2$ to the value that makes the numerator vanish. Therefore

$$
u_{c}=\frac{1}{2}, \quad \text { if } \frac{3}{4}<\alpha \bar{W}_{i c}<\frac{25}{32}
$$

$\alpha \bar{W}_{i c}$ cannot exceed 25/32, which is the maximum value of $(5 / 2) u-2 u^{2}$. Now $\theta$ decreases weakly in the underdense layer. Note that the Mach number $M \equiv u / \theta^{1 / 2}$, at the exit of the deflagration layer $\left(\eta-1 \gg \eta_{c}-1\right)$, grows with $\alpha \bar{W}_{i c} ; M$ reaches unit at $\alpha \bar{W}_{i c}=3 / 4$, and $(5 / 3)^{1 / 2}$ at $\alpha \bar{W}_{i c}=25 / 32$.

For the outer solution (9) and (10) become

$$
\begin{gathered}
\frac{1}{2} u^{2}+\frac{5}{2} \theta=\bar{W}_{i}\left[1-(1-\alpha) \bar{W}_{i c}^{2} / \bar{W}_{i}^{2}\right] \\
\mathrm{d} \bar{W}_{i} / \mathrm{d} \eta=\sigma \bar{W}_{i} / \eta^{4} u^{3 / 2} \theta^{3 / 2}\left(u-u_{c} / \eta^{2}\right)^{1 / 2} .
\end{gathered}
$$

For $\alpha$ and $\bar{W}_{i c}$ given and such that $\alpha \bar{W}_{i c}<25 / 32$, we will have $M^{2}=5 / 3$ somewhere; from (8), (A.9) and (A.10) we find that for the solution to be univalued at this (isentropic) sonic point we must have

$$
\frac{\theta^{5 / 2} \eta^{3}}{\sigma}=\frac{2 \bar{W}_{i}^{2}+(1-\alpha) \bar{W}_{i c}^{2}}{5 \bar{W}_{i}^{2}-(1-\alpha) \bar{W}_{i c}^{2}} \frac{1}{\left(1-u_{c} / u \eta^{2}\right)^{1 / 2}} \text { at } \frac{u^{2}}{\theta}=\frac{5}{3} .
$$

This condition determines $\sigma$, and then $\bar{W}_{i \infty}$ :

$$
\sigma=F_{4}\left(\alpha, \bar{W}_{i c}\right), \quad \bar{W}_{i \propto c}=F_{5}\left(\alpha, \bar{W}_{i c}\right), \quad 0<\alpha \bar{W}_{i c}<\frac{25}{32} .
$$

For $\alpha \bar{W}_{i c} \rightarrow 0$, we have $F_{4} \rightarrow 0.201, F_{5} \rightarrow 1.47$. For $\alpha \bar{W}_{i c} \rightarrow 25 / 32$ we obtain $\sigma \rightarrow 0.0297 \alpha /(2-\alpha)$; also $\bar{W}_{i \infty} \rightarrow 4.02,2.08$ and 0.996 , for $\alpha=0.2,0.4$ and 1 , respectively.

For $\bar{W}_{i c}=25 / 32 x, \sigma$ may have any value. Solving (8), (A.9) and (A.10), we get

$$
\bar{W}_{i \infty}=F_{6}(x, \sigma), \quad 0<\sigma<0.0297 \alpha /(2-x) .
$$

For $\sigma \rightarrow 0(\hat{V} \rightarrow 0)$ we find $F_{6} \rightarrow 25 / 32 x$, now there is no bremsstrahlung absorption, and we have the deflagration resuits of Paper $\mathbb{I}$.

\section{REFERENCES}

Afanas'ev Yu. V., Gamalin E. G., Krokhin O. N. and Rozanov V. B. (1977) Soviet Phys. JETP 44, 311. Ahlstrom H. G. (1982) Laser Plasma Interaction (edited by Balian Roger and Adam Jean-Claude). North-Holland, Amsterdam.

CRAXTON R. S. and MCCRORY R. L. (1980) University of Rochester, Laboratory for Laser Energetics Report 108.

Eliezer S. (1982) Laser Plasma Interaction (edited by Baliax Roger and Adam Jean-Claude). NorthHolland, Amsterdam.

Garbai-Labaune C., Fabre E., Fabbro R., Amiranoff F. and Weinfeld M. (1979) Rapport d'Activité 1979, Greco Interaction Laser-Matiere (Ecole Polytechnique, Palaiseau, France) pp. 64-75.

Gitomer, S. J., Morse R. L. and Newberger B. S. (1977) Physics Fluids 20, 234.

HaAs R. A., Mead W. C., Kruer W. L., Phillion D. W., Kornblum H. N., Linde J. D., Macquigg D., Rupert V. C. and Tirsell K. G. (1977) Physics Fluids 20, 322.

Johnston T. W. and Dawson J. M. (1973) Physics Fluids 16, 722

Max C. E., McKee C. F. and Mead W. C. (1980) Physics Fluids 23, 1620.

Mead W. C., Campbell E. M., Estabrook K. G., Turner R. E., Kruer W. L., Lee P. H. Y., Prlett B., Rupert V. C., Tirsell K. G., Stradling G. L., Ze F., Max C. E., Rosen M. D. and Lasinski B. F. (1983) Physics Fluids 26, 2316.

MORa P. (1982) Physics Fluids 25, 1051.

Nishimura H., Matsuoka F., Yagi M., Yamada K., Nakal D., McCall G. H. and Yamanaka C. (1983) Physics Fluids 26, 1688.

Ripin B. H., Decoste R., Obenschain S. P., Bodner S. E., Mclean E. A., Young F. C., Whitlock R. R., Armtrong C. M., Grun J., Stamper J. A., Gold S. H., Naged D. J., Lehmberg R. H. and McMahon J. M. (1980) Physics Fluids 23, 1012; (1981) Physics Fluids 24, 990.

SANMARTIN J. R., Ramis R., MONTañes J. L. and SANZ J.(1982). Proceedings of 1982 International Conference on Plasma Physics, Goteborg, to be published in Physics Fluids. 
Sanz J., Liñan A., Rodriguez M. and Saxmartin J. R. (1981) Physics Fluids 24, 2098.

Sanz J. and SanmartiN J. R. (1983) Physics Fluids 26, 3361.

SPITZER L. (1962) Physics of Fully Ionized Gases. Wiley, New York.

YaAkobi B., Delettrez J., Goldmax L. M., McCrory R. L., Marjoribanks R., Richardson M. C., Shvarts D., Skupsky S., Solres J. M., Verdon C., Villeneuve D. M., Boehly T., Hutchisson R. and Letzring S. (1984) Physics Fluids 27(2), 516. 